\title{
Whistleblowing in der radiologischen Praxis
}

\section{Whistleblowing als gesellschaftliches Phänomen}

Whistleblowing betrifft zumeist Missstände von erheblicher Tragweite in größeren Unternehmen, der öffentlichen Verwaltung oder Verbänden; es geht in der Regel um einen Vorfall von besonderer Bedeutung. Der Whistleblower will die von ihm identifizierten oder vermuteten Missstände aufdecken und auf ihre Beseitigung hinwirken. Seine Motive sind häufig moralisch idealistischer/altruistischer Art, sie können aber auch egoistisch konnotiert sein oder Mobbing anderer Mitarbeiter betreffen. Whistleblowing wird gesellschaftlich unterschiedlich gewürdigt, einerseits kritisiert und attackiert, andererseits begrüßt und gefördert.

\section{Ansatzpunkte für Whistleblowing in der radiologischen Praxis}

Für eine kleinere Arztpraxis dürfte Whistleblowing kein relevantes Phänomen sein, für größere Praxen oder Praxisverbünde - hierzu zählen viele radiologische Praxen - gestaltet sich die Sachlage anders. Das liegt daran, dass radiologische Praxen in einem regulatorischen Umfeld tätig sind, das verschiedentliche Ansatzpunkte für Whistleblowing bietet. Damit sind nicht nur die vertragsarztrechtlichen Anforderungen an eine den Bestimmungen von Einheitlichem Bewertungsmaßstab (EBM) und Honorarverteilungsmaßstab (HVM) entsprechende Abrechnung gegenüber der zuständigen Kassenärztlichen Vereinigung (KV) gemeint. Vielmehr können auch Beschaffungsvorgänge betroffen sein - in der Vergangenheit erregte insbesondere das Stichwort „Kontrastmittel“ - darüber hinaus Regelungsgegenstände der Qualitätssicherung in Gestalt möglicherweise fehlender Genehmigungen für bestimmte qualitätsgesicherte Leistungen, die Rahmenbedingungen der Leistungserbringung als solcher (z. B. in Gestalt der Einhaltung bestimmter Leitlinien), das Verhalten gegenüber Patienten und Mitarbeitern oder auch Anforderungen an die Vertragsgestaltung größerer Zusammenschlüsse, sei es als MVZ oder Berufsaus- übungsgemeinschaft (Stichwort: „verkappter“ Angestellter).

Für den Arbeitnehmer einer radiologischen Praxis, der einen tatsächlichen oder vermeintlichen Missstand identifiziert hat, stellen sich Fragen: Soll er den Arbeitgeber informieren (dem er möglicherweise nicht traut), soll er sich an die KV oder Ärztekammer wenden, an die Medien oder gar an die Staatsanwaltschaft? Welche Auswirkungen hat sein Vorgehen auf seine Stellung in der Praxis oder für seine berufliche Zukunft? Ist sein Verdacht überhaupt begründet, muss er befürchten wegen Falschaussage oder Verleumdung ggfs. sogar strafrechtlich verfolgt zu werden, wie kann er sich absichern?

Für den Arbeitgeber stellen sich ebenfalls Fragen: Wie soll man sich gegenüber einem Whistleblower verhalten? Welche arbeitsrechtlichen Maßnahmen sind möglich und sinnvoll? Wie vermeidet man negative Medienberichterstattung und Auswirkungen auf Patienten und Mitarbeiter?

\section{Whistleblowing in der Rechtspraxis}

Whistleblowing spart Arztpraxen nicht aus. Dies beweist ein Urteil des Landesarbeitsgerichts (LAG) Berlin-Brandenburg vom 6.11.2020, Az.: 9 Sa 426/20, welches die Anforderungen an das Verhalten eines Whistleblowers definiert und zeigt, welche Fehler der von Whistleblowing betroffene Arbeitgeber begehen kann.

\section{Sachverhalt des Urteils des Landesarbeitsgerichts Berlin-Brandenburg}

Den Kern des vorliegenden Sachverhalts bildete die Frage, ob bei einer bestimmten Untersuchung die Leitlinien für die Überwachung einer Sedierung mittels Propofol eingehalten worden waren. In der betreffenden Praxis hatten die medizinischen Fachangestellten, die über eine Weiterbildung für die Sedierung von Patienten mit Propofol verfügten, gekündigt. Die mit der Durchführung der entsprechenden Untersuchungen befasste Fachärztin wandte sich - vor

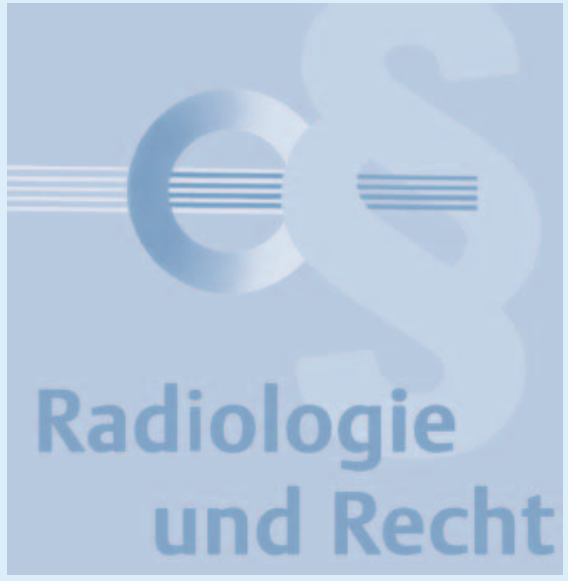

Wirksamwerden der Kündigungen - an die Geschäftsführung der Praxis:

„Wie bereits telefonisch und persönlich besprochen, möchte ich Sie nochmals darüber in Kenntnis setzen, dass nach dem Weggang der medizinischen Fachangestellten kein für die Sedierung mit Propofol entsprechend sach- und fachkundig ausgebildetes Assistenzpersonal für meine Untersuchungen mehr zur Verfügung steht. [...] Ich habe große Bedenken hinsichtlich des Patientenwohls und meiner damit verbundenen Haftung. Aus ethischer und rechtlicher Verantwortung meinen $\mathrm{Pa}$ tienten gegenüber möchte ich Sie somit um (eine gemeinsame) Entwicklung einer Lösung bitten.“

In diesem Zusammenhang verwies die Fachärztin auf eine Empfehlung der Bundesärztekammer zur Qualitätssicherung, wonach der die betreffende Untersuchung durchführende Arzt dafür verantwortlich ist, dass mitwirkendes Assistenzpersonal eine fachspezifische Qualifikation besitzt.

In der Folgezeit führte ein anderer Facharzt des Arbeitgebers bei den von der Fachärztin vorgenommenen Untersuchungen die Sedierung durch, wobei er teilweise den Raum verließ. Daraufhin nahm die Fachärztin telefonisch Kontakt mit dem Justiziar der zuständigen Ärztekammer auf, um die Rechtslage zu klären. In der Folgezeit teilte der Arbeitgeber der Fachärztin mit, die Anwesenheit eines weiterer Facharztes sei bei den Untersuchungen nicht länger erforderlich, weil das Assistenzpersonal mittlerweile hinreichend erfahren sei. Die Fachärztin widersprach und teilte ihrem Arbeitgeber mit, sie habe telefonischen 
Kontakt mit der zuständigen Ärztekammer aufgenommen. Ferner wandte sich die Fachärztin erneut an die Ärztekammer und bat um eine schriftliche Auskunft zur Rechtslage und um strikte Diskretion, weil ihr von Seiten des Arbeitgebers bereits ernste Konsequenzen aufgrund einer Einschaltung der Ärztekammer angedroht worden seien.

Die Ärztekammer forderte daraufhin den Arbeitgeber unter Übersendung des Schreibens der Fachärztin zur Stellungnahme auf. Der Arbeitgeber teilte der Ärztekammer mit, die Facharztstandards bei Diagnostik und Therapie würden in der Praxis eingehalten; einen etwaigen Verstoß hätte die Fachärztin als Leiterin des Arbeitsbereichs im Übrigen selbst zu vertreten.

Gegenüber der Fachärztin erklärte der Arbeitgeber in der Folge die Kündigung des Arbeitsverhältnisses. Diese habe eine nicht mit dem Arbeitgeber abgestimmte Rechtsauskunft der Ärztekammer zu praxisinternen Vorgängen eingeholt. Dies begründe eine schwere Störung des arbeitsvertraglichen Vertrauensverhältnisses, zudem hätte der Arbeitgeber unverzüglich nach Erhalt der Auskunft hierüber informiert werden müssen. Weiterhin habe die Fachärztin nicht nur die Ärztekammer, sondern auch eine Mitarbeiterin der Fachgesellschaft über interne Betriebsabläufe der Praxis informiert und diese unzutreffend in negativer Art und Weise dargestellt.

Die Fachärztin hat hiergegen Kündigungsschutzklage erhoben und beantragt festzustellen, dass das Arbeitsverhältnis nicht durch die Kündigung des Arbeitgebers beendet wurde, sondern fortbesteht.

\section{a. Erste Instanz: Kündigung rechtmäßig}

Das Arbeitsgericht Berlin hat die Kündigung zunächst bestätigt. Die Fachärztin habe ihre Rücksichtnahmepflichten gegenüber ihrem Arbeitgeber verletzt. Es sei einerseits zu berücksichtigen, ob einem Arbeitnehmer andere wirksame Mittel zur Verfügung standen, um etwas gegen den angeprangerten Missstand zu tun, andererseits aber ob ein öffentliches Interesse an einer Offenlegung der Information bestand. Ausgehend hiervon sei die Information der Ärztekammer eine unangemessene Reaktion auf eine möglicherweise rechtswidrige Durchführung von Untersuchungen in der Praxis des Arbeitgebers gewesen. Eine etwaige Verletzung von Leitlinien sei keine Verletzung von Berufspflichten. Der Fachärztin sei eine gemeinsame Klärung der möglichen Rechtswidrigkeit der Durchführung der Untersuchungen zumutbar gewesen, wofür bei der Ärztekammer Schlichtungsausschüsse bestünden, deren Einschaltung nicht zu einem berufsrechtlichen Ermittlungsverfahren seitens der Ärztekammer führe. Auch wenn die Ärztin nach ihrem Vortrag keine Anzeige erhoben und um Diskretion gebeten habe, habe die Ärztekammer als Körperschaft des öffentlichen Rechts einen solchen Sachverhalt zu ermitteln, ohne diesbezüglich an Anweisungen gebunden zu sein. In der Abwägung der beiderseitigen Interessen überwiege daher das Interesse des Arbeitgebers an einer Beendigung Arbeitsverhältnisses.

\section{b. Zweite Instanz: Kündigung rechtswidrig}

Das Landesarbeitsgericht Berlin-Brandenburg hat im Rahmen des Berufungsverfahrens die Kündigung des Arbeitgebers als rechtswidrig angesehen und wichtige Kriterien für das Verhalten von Whistleblower und Arbeitgeber herausgearbeitet.

\section{aa. Keine wissentlich unwahren oder leichtfertig falschen Angaben des Whistleblowers}

Eine Anfrage einer Ärztin an die Ärztekammer zur Bewertung ärztlichen Vorgehens ohne Angabe wissentlich unwahrer oder leichtfertig falscher Angaben ist nach Auffassung des LAG regelmäßig keine Verletzung von Rücksichtnahmepflichten, die für sich genommen eine Kündigung rechtfertigen könnte. Dies gilt auch dann, wenn diese Anfrage aufgrund der Begründung von der Ärztekammer im Sinne einer Beschwerde oder Anzeige weiterbearbeitet wird.

Insofern stützt sich das LAG auf die Rechtsprechung des Bundesarbeitsgerichts zur Einschaltung der Staatsanwaltschaft durch einen Arbeitnehmer wegen eines vermeintlich strafbaren Verhaltens des Arbeitgebers oder seiner Repräsentanten. Danach ist die Einschaltung der Staatsanwaltschaft als Wahrnehmung staatsbürgerlicher Rechte soweit nicht wissentlich unwahre oder leichtfertig falsche Angaben gemacht werden - im Regelfall keine eine Kündigung rechtfertigende Pflichtverletzung. Unter denselben Voraussetzungen ist - so folgert das LAG - auch die Einschaltung der Ärztekammer als Wahrnehmung ärztlicher Verantwortung zulässig. Der Ärztekammer obliege die Wahrnehmung beruflicher Belange ihrer Angehörigen unter Beachtung der Interessen der Allgemeinheit und die Überwachung der Erfüllung der Berufspflichten der Kammerangehörigen und der Berufsangehörigen.

Darüber hinaus sei eine Einschaltung der Ärztekammer auch im Falle eines Konfliktes zwischen Ärzten zulässig und nicht zwangsläufig als Anzeige eines Fehlverhaltens zu verstehen. Es handele sich bei der Ärztekammer nicht um eine Institution wie die Staatsanwaltschaft, deren Aufgabe allein in der Strafverfolgung liege. Vielmehr obliege der Ärztekammer auch die Wahrnehmung der Interessen jedes einzelnen Mitglieds und, wie sich auch an der vorgesehenen Einrichtung von Schlichtungsausschüssen ergibt, die Schlichtung von Streitigkeiten, die sich aus dem Berufsverhältnis ergeben. Im Hinblick auf dieses Ziel einer internen Klärung von Konflikten zwischen Ärzten stelle eine Anfrage an die Ärztekammer als der eigenen berufsständischen Organisation mit dem Ziel einer internen Klärung unter Ärzten, soweit nicht wissentlich unwahre oder leichtfertig falsche Angaben gemacht werden, keine Verletzung von Rücksichtnahmepflichten dar.

Doch wann handelt es sich um wissentlich unwahre oder leichtfertig falsche Angaben eines Whistleblowers?

Zunächst: Legt der Whistleblower in seiner Anfrage offen, auf welche Annahmen sich seine Anfrage stützt und dass es sich um seine - subjektive - Bewertung handelt, so macht der Whistleblower bereits keine wissentlich unwahren oder leichtfertig falschen Angaben.

Bei der Frage, ob man es mit wissentlich unwahren oder leichtfertig falschen Angaben des Whistleblowers zu tun hat, ist jedoch auch zu berücksichtigen, wie konkret der Arbeitgeber zu den Angaben des Whistleblowers Stellung nehmen kann. So hätte der Arbeitgeber im vorliegenden Fall der 
Angabe der Fachärztin, der andere Facharzt sei bei ihren Untersuchungen als anwesend in der Dokumentation vermerkt, in Wahrheit aber nicht in der Praxis gewesen, dem mit konkretem Vortrag zu den in der Dokumentation festgehaltenen Anwesenbzw. Abwesenheitszeiten dieses Arztes entgegengetreten können, was aber nicht geschah. Dies wertete das LAG als Indiz, dass die diesbezügliche Angabe der Fachärztin nicht als leichtfertig falsch zu bewerten sei.

Eine unterschiedliche Einschätzung von Ausbildungs- und Erfahrungsanforderungen und ein unterschiedliches Verständnis, wann von einer „eigenständigen“ Durchführung bzw. Überwachung der Sedierung durch eingesetzte Assistenzkräfte auszugehen ist, macht die Angaben nicht falsch. So führte der Arbeitgeber in seiner Stellungnahme gegenüber der Ärztekammer - entgegen der Darstellung der Fachärztin - aus, nur für die Entscheidung über die Gabe eines Sedativums bedürfe eines Zertifikats, nicht für die Überwachung der Sedierung selbst. Entscheidend ist, ob das Verständnis des Whistleblowers weder leichtfertig falsch noch gar haltlos ist, was das LAG im Fall der Fachärztin nicht feststellen konnte; zudem handele es sich bei der Ärztekammer um eine Institution, die zu einer differenzierten Bewertung unterschiedlicher ärztlicher Auffassungen in der Lage sei.

Die Bezeichnung von ärztlichen Leitlinien durch den Whistleblower als rechtlich verbindlich ist keine leichtfertig falsche Angabe. Die Bezugnahme auf die Leitlinien charakterisiere im Fall der Fachärztin - so das LAG - nur die konkreten Bedingungen der Arbeit und die Frage, ob damit auch im Hinblick auf hierzu vorliegende Leitlinien und sonstige Vorgaben eine Tätigkeit unter Einhaltung der Regeln ärztlicher Kunst gegeben sei. Von einem Whistleblower könnten keine besonderen Kenntnisse dazu verlangt werden, wie sich ein behaupteter Normverstoß in das Gesamtgefüge einer Rechtsmaterie einordne.

Auch eine in vorwurfsvollem Ton formulierte Anfrage verletzte nicht die Rücksichtnahmepflichten des Whistleblowers gegenüber seinem Arbeitgeber, solange diese sachbezogen bleibe. Im Fall der Fachärztin sei zu berücksichtigen, dass diese nach ihrem Hinweis ohne vorheriges Gespräch über eine etwa anstehende Beendigung des $\mathrm{Ar}$ beitsverhältnisses eine Kündigung erhalten und insoweit ihrerseits keine rücksichtsvolle Behandlung erfahren habe, die wiederum eine besondere Rücksichtnahme ihrerseits nahelegen würde.

\section{bb. Interne Klärung in der Regel vorrangig}

Der Whistleblower hat, soweit dies zumutbar ist, Hinweise auf ein rechtswidriges Verhalten in erster Linie gegenüber Vorgesetzten vorzubringen. Diese Vorgabe einer im Rahmen des Zumutbaren zunächst zu versuchenden internen Klärung vor einer Einbeziehung weiterer Stellen als Ausfluss von Rücksichtnahmepflichten gilt auch für eine Anzeige bei der Ärztekammer. Auch wenn eine solche gegenüber beispielsweise einer Information der Öffentlichkeit ein deutlich milderes Mittel darstellt, kann auch die Ärztekammer Sanktionen verhängen. Entsprechend ist, auch unter dem Vorbehalt der Zumutbarkeit, zuvor die Möglichkeit einer Abhilfe einzuräumen.

Im vorliegenden Fall hielt das LAG die Bemühungen der Fachärztin für ausreichend. Sie habe nicht nur die aus ihrer Sicht bestehenden Probleme geschildert, sondern auch Möglichkeiten der Abhilfe dargelegt, letzteres nicht nur im Sinne einer praktisch schwer umsetzbaren sofortigen Einstellung geeigneten Personals, sondern auch in Form von konkreten Vorschlägen zur Fortbildung der Beschäftigten und der Änderung organisatorischer Abläufe bis zum Abschluss derselben.

Schließlich sei es ein Unterschied, ob direkt Anzeige erstattet werde oder das Problem im Wege einer Anfrage an die Ärztekammer herangetragen werde. Die Fachärztin habe damit die Klärung eines Konfliktes unter Ärzten herbeiführen und keine disziplinarischen Maßnahmen auslösen wollen. Die Bitte um eine vertrauliche Behandlung zeige, dass keine Einschaltung weiterer Stellen oder weitere Maßnahmen angestrebt wurden. Die Schilderung des zugrunde liegenden Sachverhalts und der Konfliktlage aus der Sicht der Fachärztin mache die Anfrage nicht zur Anzeige. Ohne Schilderung des Sachverhalts könne die Frage, wie sich die Fachärztin im Hinblick auf die ihr obliegenden Berufspflichten verhalten solle, nicht beantwortet werden.

\section{cc. Keine Schädigungsabsicht des Whistleblowers}

Den Vorwurf des Arbeitgebers, die Fachärztin habe durch eine Information des Personals über die aus Sicht der Fachärztin erforderliche Einhaltung von Leitlinien für die Kündigung einer Mitarbeiterin gesorgt, woraus sich ergebe, dass die Anfrage an die Ärztekammer letztlich in Schädigungsabsicht erfolgt sei, hält das LAG für irrelevant. Die Kenntnis einschlägiger Leitlinien zur Durchführung einer bestimmten ärztlichen Maßnahme gehöre zu den zumindest wünschenswerten Kenntnissen des eingesetzten Personals. Soweit die Überwachung des Patienten auf Mitarbeiterinnen übertragen werde, sollten sich diese auch der damit verbundenen Verantwortung bewusst sein. Darüber hinaus liege gerade keine Information der Öffentlichkeit vor, die bei einer etwaigen Schädigungsabsicht als solche sicherlich geeignet gewesen wäre, den weiteren Erfolg der Praxis zu beeinträchtigen. Auch aus dem Gespräch der Fachärztin mit einer Mitarbeiterin der Fachgesellschaft könne keine Schädigungsabsicht hergeleitet werden.

Die im Arbeitsverhältnis bestehenden Rücksichtnahmepflichten beinhalten auch, während des Bestandes des Arbeitsverhältnisses Betriebs- und Geschäftsgeheimnisse zu bewahren. Eine Verletzung dieser Verpflichtung kann abhängig von den Umständen des Einzelfalls eine Kündigung rechtfertigen, insbesondere wenn anzunehmen ist, der Arbeitnehmer werde den Arbeitsvertrag auch zukünftig in gleicher oder ähnlicher Weise verletzen. Entsprechendes gilt für bewusst wahrheitswidrig aufgestellte Tatsachenbehauptungen, etwa wenn sie den Tatbestand der üblen Nachrede erfüllen. Eine Verletzung dieser Pflichten durch die Fachärztin sah das LAG jedoch nicht. Allein die Tatsache, dass die Fachärztin sich als Mitglied der Fachgesellschaft mit einer Mitarbeiterin der Fachgesellschaft ausgetauscht habe, sei nicht zu beanstanden. Dies gelte auch für einen Austausch betreffend Erfahrungen aus der Praxis und darüber, ob Leitlinien und Standards, wie sie u. a. von dieser Gesellschaft entwickelt werden, in der Praxis Akzeptanz finden oder in Frage gestellt werden. Auch wenn bekannt ist, wer wo arbeitet, sei es aufgrund persönlicher Bekanntschaft, aufgrund üblicher 
Kenntnisse in Fachkreisen oder aufgrund im Internet zugänglicher Informationen, schließe der dann für Dritte erkennbare jeweilige Erfahrungshintergrund für Äußerungen einen Gedankenaustausch nicht aus.

Selbst wenn man annehme, die Fachärztin habe in diesem Gespräch Erklärungen zu betrieblichen Abläufen abgegeben, die die Grenzen zulässigen Austauschs überschreiten, würde auch dies keine Kündigung ohne vorhergehende einschlägige Abmahnung rechtfertigen. Einer Abmahnung bedürfe es nach Maßgabe des Verhältnismäßigkeitsgrundsatzes allein dann nicht, wenn bereits ex ante erkennbar sei, dass eine Verhaltensänderung auch nach Ausspruch einer Abmahnung nicht zu erwarten oder die Pflichtverletzung so schwerwiegend sei, dass selbst deren erstmalige Hinnahme durch den Arbeitgeber nach objektiven Maßstäben unzumutbar und offensichtlich - auch für den Arbeitnehmer erkennbar - ausgeschlossen sei. Diese Voraussetzungen seien im Fall der Fachärztin nicht gegeben. Soweit hier Grenzen verletzt worden sein sollten, gäbe es keine Anhaltspunkte dafür, dass die Fachärztin nicht bereit wäre, im Falle einer Abmahnung bei künftigen Anfragen und Erörterungen jegliche Äußerungen über konkrete interne Vorgänge zu unterlassen.
Schließlich sei auch kein provokatives oder aggressives Verhalten der Fachärztin dergestalt festzustellen, dass sie die Kündigung durch den Arbeitgeber provoziert habe. Das Vertreten eines anderen ärztlichen Standpunktes zur fachgerechten Behandlung von Patienten und eine geforderte Einhaltung diesbezüglicher Leitlinien ist keine Provokation einer Kündigung.

\section{Fazit: Rechtsfragen des Whistleblowings gewinnen an Bedeutung}

Whistleblowing findet allmählich Eingang in die Rechtswirklichkeit, das zeigt das vorliegende Urteil des LAG Berlin-Brandenburg. Zudem muss die Bundesrepublik Deutschland die EU-Richtlinie zum Schutz von Personen, die Verstöße gegen das Unionsrecht melden, bis zum 17. Dezember 2021 umsetzen. Ein Gesetzentwurf ist an unterschiedlichen Vorstellungen von CDU/ CSU und SPD bislang gescheitert.

Wie der vorliegende Fall verdeutlicht, birgt Whistleblowing sowohl für den Whistleblower als auch den Arbeitgeber erhebliche Risiken. Für den Whistleblower geht es um den Bestand seines Arbeitsverhältnisses, möglicherweise um seine berufliche $\mathrm{Zu}$ - kunft. Er darf keine wissentlich unwahren oder leichtfertig falschen Angaben machen, ihn treffen Rücksichtnahmepflichten, er muss also die Möglichkeit zur Abhilfe schaffen und sich genau überlegen, welche Information er an wen „nach außen“ gibt.

Für die Bewertung des Verhaltens des Whistleblowers kommt es aber auch darauf an, welche Strukturen der Arbeitgeber zur Verfügung stellt, um eine Information des Whistleblowers über vermeintliche oder tatsächliche Missstände so zu ermöglichen, dass die Angaben verifizierbar sind, die Anonymität des Whistleblowers aber gewahrt bleibt.

Nach dem Entwurf des Hinweisgeberschutzgesetzes sollen - mit einer Übergangsfrist von zwei Jahren - Unternehmen mit mehr als 50 Mitarbeitern verpflichtet werden, eine Whistleblower-Hotline einzurichten. Es bestehen also gewichtige Gründe, sich - auch in der radiologischen Praxis - näher mit dem Thema „Whistleblowing“ zu beschäftigen.

Dr. Horst Bonvie
Rechtsanwalt
Fachanwalt für Medizinrecht
Rechtsanwälte Wigge
Großer Burstah 42
20457 Hamburg

